OPEN ACCESS

Edited by:

Wei Luo,

Jiangnan University, China

Reviewed by:

Jingyu Wang,

Westlake Institute for Advanced Study

(WIAS), China

Yun Chen,

Chalmers University of Technology,

Sweden

*Correspondence:

Mingdong Yao

mingdong.yao@tju.edu.cn

${ }^{\dagger}$ These authors share first authorship

Specialty section: This article was submitted to

Synthetic Biology,

a section of the journal

Frontiers in Bioengineering and

Biotechnology

Received: 30 June 2020

Accepted: 18 August 2020

Published: 04 September 2020

Citation:

Song T, Wu N, Wang C, Wang Y,

Chai F, Ding M, Li X, Yao M, Xiao W and Yuan Y (2020) Crocetin

Overproduction in Engineered Saccharomyces cerevisiae via Tuning Key Enzymes Coupled With Precursor

Engineering.

Front. Bioeng. Biotechnol. 8:578005.

doi: 10.3389/fbioe.2020.578005

\section{Crocetin Overproduction in Engineered Saccharomyces cerevisiae via Tuning Key Enzymes Coupled With Precursor Engineering}

\author{
Tianqing Song ${ }^{1,2+}$, Nan Wu ${ }^{1,2+}$, Chen Wang ${ }^{1,2}$, Ying Wang ${ }^{1,2}$, Fenghua Chai ${ }^{1,2}$, \\ Mingzhu Ding ${ }^{1,2}$, Xia Li ${ }^{1,2}$, Mingdong Yao ${ }^{1,2 *}$, Wenhai Xiao ${ }^{1,2}$ and Yingjin Yuan ${ }^{1,2}$
}

\begin{abstract}
'Frontier Science Center for Synthetic Biology, Key Laboratory of Systems Bioengineering (Ministry of Education), School of Chemical Engineering and Technology, Tianjin University, Tianjin, China, ${ }^{2}$ Collaborative Innovation Center of Chemical Science and Engineering (Tianjin), Tianjin University, Tianjin, China
\end{abstract}

Crocetin, an important natural carotenoid dicarboxylic acid with high pharmaceutical values, has been successfully generated from glucose by engineered Saccharomyces cerevisiae in our previous study. Here, a systematic optimization was executed for crocetin overproduction in yeast. The effects of precursor enhancement on crocetin production were investigated by blocking the genes involved in glyoxylate cycle [citric acid synthase (CIT2) and malic acid synthase (MLS1)]. Crocetin titer was promoted by $50 \%$ by $\Delta$ ClT2 compared to that of the starting strain. Then, the crocetin production was further increased by $44 \%$ through introducing the forward fusion enzymes of PsCrtZ (CrtZ from Pantoea stewartii)-CsCCD2 (CCD2 from Crocus sativus). Consequently, the crocetin titer reached to $1.95 \pm 0.23 \mathrm{mg} / \mathrm{L}$ by overexpression of PsCrtZ-CsCCD2 followed by medium optimization. Eventually, a titer of $12.43 \pm 0.62 \mathrm{mg} / \mathrm{L}$ crocetin was achieved in $5-\mathrm{L}$ bioreactor, which is the highest crocetin titer reported in microorganisms.

Keywords: metabolic engineering, crocetin, enzyme fusion, precursor engineering, Saccharomyces cerevisiae

\section{INTRODUCTION}

Crocetin, a carotenoid derivative compound isolated from the stigma of Crocus sativus L (Saffron), has great potential in the clinical treatment of cardiovascular and neuropsychological diseases (such as hypertension, atherosclerosis, and depression) (Nassiri-Asl and Hosseinzadeh, 2015; Xiang et al., 2017), and even in cancer therapy (Bathaie et al., 2013; Moradzadeh et al., 2018). Due to the limitations of saffron resources and the lower yield of traditional extraction, the output of crocetin is far from the market demand. De novo biosynthesis of crocetin in yeast (Chai et al., 2017) and Escherichia coli (Wang et al., 2019) has been achieved, respectively, but the titers were still quite low. Learning from previous results, two main clues for further optimization are enriching precursor pools and tuning limited metabolic reaction step (conversion zeaxanthin to crocetin dialdehyde by CCD).

On one hand, the de novo crocetin biosynthesis pathway in Saccharomyces cerevisiae is relatively long and consecutive steps comprised the mevalonate pathway (MVA) as precursor pool and the following heterologous crocetin formation pathway (Figure 1A). Thus, to enhance the MVA 
pathway flux is also crucial for final crocetin production. Previous efforts to engineer MVA pathway relevant genes to increase acetyl-CoA and MVA levels have achieved significant success in boosting terpenoid yield and productivity in S. cerevisiae (Ro et al., 2006; Lv et al., 2016). At the same time, it should be noted that acetyl-CoA used for terpenoids production is in the cytoplasm. Acetyl-CoA in the peroxisomes and cytosol could be converted to $\mathrm{C}_{4}$ organic acids (malate and succinate) via the glyoxylate cycle (GYC) (Figure 1A), that will be transferred to the mitochondria for oxidation by malic enzyme and the tricarboxylic acid (TCA) cycle (Chen et al., 2013). Previous studies had revealed that deleting the related proteins of the GYC [i.e., citric acid synthase (CIT2) in peroxidase body and malic acid synthase (MLS1) in the cytoplasm] was to decrease the digestion of acetyl-CoA in the cytoplasm to be used for the TCA cycle (Cobine et al., 2012; Chen et al., 2013; Krivoruchko et al., 2015). Thus, in order to further enhance precursor pools, deleting related genes to avoid acetyl-CoA entering GYC cycle and to prevent FPP consumption by dephosphorylating would be a good choice.

On the other hand, efforts in protein fusion formed by linker have been widely used to promote heterologous production of versatile products in micro-organisms (Deng et al., 2016). Zhou et al. (2012) reported that miltiradiene production was significantly improved and byproduct accumulation was reduced by the fusion of copalyl diphosphate synthase $(\mathrm{Sm})$ and kaurene synthase-like (SmKSL) from Salvia miltiorrhiza, as well as the fusion of GGPP synthase (BTS1) and farnesyl diphosphate synthase (ERG20). In addition to linker type (Sabourin et al., 2007) and linker length (Zhao et al., 2016), fusion orientation (forward or reverse) could significantly affect the performance of fusion protein. Lee et al. (2016) pointed out fusing a coumarate-CoA ligase (4CL) and benzalace-tone synthase from Rheum palmatum (RpBAS) in the 4CL-RpBAS orientation gave significant improvement on final raspberry ketone levels, but the reverse version (RpBAS-4CL) did not work. In our previous work, it was found that the fusion orientation of geraniol synthase (GES) and ERG20 provided no significant effects on geraniol production in S. cerevisiae (Jiang et al., 2017). In our current case, since CCD2 has a more hydrophobic substrate channel on N-terminal (Chai et al., 2017), and CrtZ is just responsible for zeaxanthin formation (which is the substrate of CCD2), the fusion of CrtZ and CCD2 would enhance the substrate accessibility of CCD2, consequently leading to higher zeaxanthin conversion. And because the previously available structural study of CCDs revealed that a striking "windmill" architecture consisting of seven-bladed $\beta$-propeller was highly conserved on the C-terminal, thus it is also worth to explore the fusion orientation of $\mathrm{CrtZ}$ and CCD2 in the following work for further promoting crocetin level.

In present work, the crocetin production was further improved by precursor engineering coupled with tuning key enzymes. Firstly, two genes CIT2 and MLS1 that consuming cytoplasm acetyl-CoA were individually knocked out. The corresponding results demonstrated that crocetin titer was enhanced by $50 \%$ with CIT2 knockout. Secondly, a synthetic fusion protein of CrtZ and CCD2 was constructed for unlocking the limiting step and the effect of fusion protein orientation was also investigated. Protein structural simulation revealed that the fusion orientation would have significant influence in the catalytical activity of fusion protein of CrtZ and CCD2. And the forward fusion enzymes of PsCrtZ-CsCCD2 promoted the crocetin titer by $44 \%$, which just matched the protein structural simulation results. Finally, a titer of $12.43 \pm 0.62 \mathrm{mg} / \mathrm{L}$ crocetin was obtained by medium optimization and 5 -L fedbatch fermentation, which was the highest crocetin titer reported in micro-organisms so far. This study not only completely reproduces the classic metabolic engineering research loop from precursor engineering to enzyme engineering and process optimization, but also highlights the synergy between precursor engineering and key enzymes engineering is important to achieve microbial overproduction of natural products.

\section{MATERIALS AND METHODS}

\section{Strains Cultivation}

All the yeast strains used in this study were listed in Table $\mathbf{1}$. For shake-flask cultivation, colonies were picked from solid plates and cultured in $3 \mathrm{~mL}$ SC medium (Chai et al., 2017) for overnight growth at $30^{\circ} \mathrm{C}$. Then $500 \mu \mathrm{L}$ culture was transferred into $5 \mathrm{~mL}$ fresh SC medium for seeds growing. After nearly $15 \mathrm{~h}$ cultivation, the seed culture was inoculated into $50 \mathrm{~mL}$ YPD medium with an initial $\mathrm{OD}_{600}$ of 0.1 . For crocetin production, $1 \%(\mathrm{w} / \mathrm{v}) \mathrm{D}-(+)$-galactose was supplied into YPD medium as the inducer. The whole fermentation lasted for $96 \mathrm{~h}$ at $20^{\circ} \mathrm{C}$. In the meanwhile, samples were collected at $42 \mathrm{~h}$ and the concentration of acetyl-CoA is quantified by BioVision's PicoProbe Acetyl CoA Assay Kit (MAK039), according to the protocols provided by the manufacturer.

\section{DNA Manipulation and Strain Construction}

All the plasmids (listed in Supplementary Table S1) used in this study were constructed in E. coli DH5 $\alpha$. All the primers used in this study were presented in Supplementary Table S2. The gene knockout strains were constructed by yeast homologous recombination method. The basic gene knockout cassette was designed as left homologous arm (gene_L)-URA3right homologous arm (gene_R) and was assembled by overlap extension PCR (OE-PCR). Among these three parts, the left or right homologous arms were obtained by PCR amplification based on the sequences of CIT2, or MLS1 in Saccharomyces Genome Database (SGD). Finally, the gene knockout cassette (gene_L-URA3-gen_R) was cloned into plasmid pEASY-Blunt (generating plasmids pEASY-Blunt-Q-03,04) for sequencing verification. Yeast transformation was conducted via the LiAc/SS carrier DNA/PEG method (Gietz, 2006). Before that, these plasmids should be digested with NotI. The recombinant yeasts were selected on SC-URA (Chai et al., 2017) plates and verified by PCR. Genome integration marker $U R A 3$ was recycled through 5-FOA selection according to Boeke et al. (1984).

CrtZ and CCD2 were fused with the short flexible GGGS linker (Yu et al., 2015). In order to construct protein fusion 


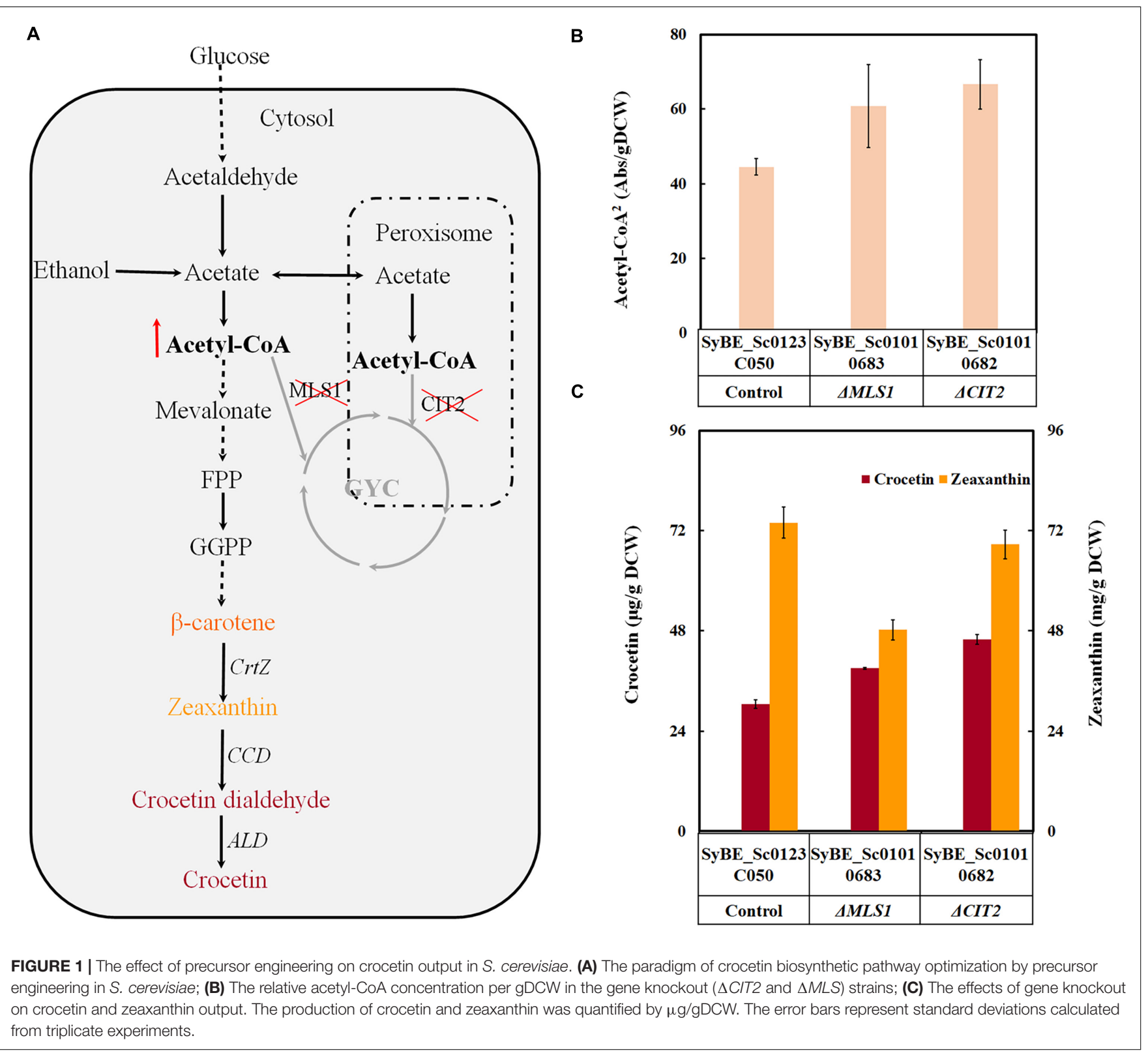

TABLE 1 | S. cerevisiae strains used in this study.

\begin{tabular}{|c|c|c|}
\hline Strain & Description & Source \\
\hline SyBE_Sc0123C050 & 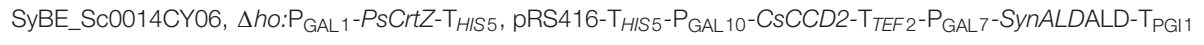 & This lab \\
\hline SyBE_Sc01010683 & SyBE_Sc0123C050, $\Delta M L S 1$ & This study \\
\hline SyBE_Sc02130062 & SyBE_Sc0014CY06, $\Delta$ ClT2, pRS416-PGAL10-PsCrtZ-CsCCD2-T TEF2-PGAL7-SynALD-TPG11 ${ }^{1}$ & This study \\
\hline SyBE_Sc02130063 & SyBE_Sc0014CY06, $\Delta$ ClT2, pRS416-PGAL 10-CsCCD2-PSCrtZ-T TEF $2-P_{\text {GAL } 7}-S y n A L D-T_{P G 11}{ }^{1}$ & This study \\
\hline
\end{tabular}

\footnotetext{
${ }^{1}$ - is for protein fusion.
}

cassettes, the promoters $\left(\mathrm{P}_{\mathrm{GAL} 1}, \mathrm{P}_{\mathrm{GAL} 7}\right.$, and $\left.\mathrm{P}_{\mathrm{GAL} 10}\right)$, and terminators $\left(\mathrm{T}_{H I S 5}, \mathrm{~T}_{T E F 2}\right.$, and $\left.\mathrm{T}_{\mathrm{PGL} 1}\right)$ were amplified from the genomic DNA of $S$. cerevisiae CEN.PK2-1C. PsCrtZ and
CsCCD2 were recovered from plasmid pUC57-Simple-05 and pUC57-Simple-10 via PCR amplification, respectively. Cassette $\mathrm{P}_{\mathrm{GAL} 10}-P_{s} C r t Z-C s C C D 2-\mathrm{T}_{T E F 2}-\mathrm{P}_{\mathrm{GAL}}-\mathrm{SynALD}-\mathrm{T}_{\mathrm{PGI} 1}$ 
and $\quad \mathrm{P}_{\mathrm{GAL} 10}-C s C C D 2-P s C r t Z-\mathrm{T}_{T E F 2}-\mathrm{P}_{\mathrm{GAL}}-\mathrm{SynALD}-\mathrm{T}_{\mathrm{PGI} 1}$ (for protein fusion) were assembled by OE-PCR. The OEPCR products were digested by BamHI/EcoRI and inserted into the corresponding sites of plasmid pRS416, obtaining the plasmid pRS416-L-04 and pRS416-L-05, respectively (Supplementary Table S1). Then the module PaL10PsCrtZ-CsCCD2-T ${ }_{T E F 2}-\mathrm{P}_{\mathrm{GAL}}$-SynALD-T $\mathrm{TGI}$ was cut from pRS416-L-04 by BamHI/EcoRI and inserted into the same site of plasmid pRS426, generating plasmid pRS426-L-01 (Supplementary Table S1). Transformation of plasmids into yeast strains was performed with the same method for gene knockout.

\section{Homology Modeling and Structural Analysis by Computational Simulation}

To explore the effect of protein fusion version of CrtZ and CCD2, the tridimensional structure models of the CsCCD2, forward fusion-protein $P s C r t Z-C s C C D 2$ and reverse fusion-protein CsCCD2-PsCrtZ were built and optimized by EasyModeller (Kuntal et al., 2010). Two reported structures from PDB (PBDID: $4 z$ ri and $3 n p e$ ) were used as the templates of $P s C r t Z$ and CsCCD2, respectively. The three modeled structures were analyzed using Pymol software (Delano and Bioinformatics, 2002).

\section{Medium Optimization at Shake Flaks Level}

Plackett-Burman (Ahuja et al., 2004) two-level fractional factorial design was used to verify the main effects among the conventional parameters including carbon source, nitrogen source and inorganic salt ions associated with yeast growth and crocetin production. $N=12$ experimental design was selected to explore the significance of seven factors including glucose, yeast extract, peptone, $\left(\mathrm{NH}_{4}\right)_{2} \mathrm{SO}_{4}, \mathrm{MgSO}_{4}, \mathrm{KH}_{2} \mathrm{PO}_{4}$, and $\mathrm{CaCl}_{2}$. Two levels' design was set for each factor, as shown in Supplementary Tables S3, S4. Then, the designed experiment was performed in shake-flask level and crocetin was extracted and quantified according to the methods described below. The regression analysis was performed accordingly, which would show priority of all the tested factors (Supplementary Table S5). The significant factors were used for further optimization by steepest ascent method in which their concentration increased successively (Supplementary Table S6). The result was used to determine the central point of the following Box-Behnken response surface methodology (RSM) optimization (Supplementary Table S7) (Song et al., 2017).

The Design-Expert software was applied for the BoxBehnken Design, and three levels were selected for each factor (Supplementary Tables S7, S8). Through regression fitting analysis, a quadratic response surface regression model was established, according to which the optimal level of response factor for crocetin production was calculable.

\section{Fed-Batch Fermentation}

The strain SyBE_Sc02130068 was used for fed-batch fermentation in 5-L bioreactor (BLBIO-5GJG-2, Shanghai, China). $100 \mu \mathrm{L}$ glycerol-stock cell culture was inoculated into
$25 \mathrm{~mL}$ SC-URA medium, culturing at $30^{\circ} \mathrm{C}, 250 \mathrm{rpm}$ for overnight growth. Then the culture was transferred to $200 \mathrm{~mL}$ fresh SC medium and cultivated $10 \mathrm{~h}$ until $\mathrm{OD}_{600}$ reached about 5.0. Then, seed cultures were transferred to the bioreactor containing $1.8 \mathrm{~L}$ fermentation medium. The $\mathrm{pH}$ and the air flow were controlled at 5.5 and $2.5 \mathrm{vvm}$, respectively. And the dissolved oxygen was maintained above $40 \%$ through cascading with the stirring speed from 400 to $600 \mathrm{rpm}$. The temperature and feeding control varied according to the fermentation stage. The starting temperature of fermentation was $30^{\circ} \mathrm{C}$, which was in favor of cell growth. As the crocetin pathway was controlled by GAL promoters $\left(\mathrm{P}_{\mathrm{GAL} 1}, \mathrm{P}_{\mathrm{GAL}}\right.$, and $\left.\mathrm{P}_{\mathrm{GAL} 10}\right)$, which were induced by galactose and inhibited by glucose. To get more cell density and higher yield, the fermentation process was divided into two stages: cell growth stage and crocetin accumulation stage. For the cell growth stage, fermentation was carried out at $30^{\circ} \mathrm{C}$ and $500 \mathrm{~g} / \mathrm{L}$ concentrated glucose solution was feeding periodically to control the glucose concentration less than $1 \mathrm{~g} / \mathrm{L}$. $10 \mathrm{~g}$ yeast extract was added into the bioreactor every $12 \mathrm{~h}$ by adding $400 \mathrm{~g} / \mathrm{L}$ concentrated yeast extract stock solution. When the cell grew into the plateau, the fermentation entered the crocetin accumulation stage. After the fermentation temperature was reduced to $20^{\circ} \mathrm{C}, 10 \mathrm{~g} / \mathrm{L}$ of $\mathrm{D}$-(+)-galactose was fed to induce crocetin formation. The feeding carbon source was changed into ethanol accordingly. The ethanol was controlled below $5 \mathrm{~g} / \mathrm{L}$ through adjusting the feeding rate according to the ethanol concentration determined by HPLC.

\section{Carotenoids Extraction and Determination}

The methods of carotenoids extraction and determination have been described previously (Chai et al., 2017). The harvested cells were broken through quick heating and cooling. The then carotenoids were extracted from the lysis solution (acetone containing $1 \%(\mathrm{w} / \mathrm{v})$ butylated hydroxytoluene). The acetone phase was evaporated by nitrogen blow and then re-dissolved for HPLC analysis. To measure $\beta$-carotene and zeaxanthin, samples were dissolved in acetone and detected at $450 \mathrm{~nm}$. In the meanwhile, for crocetin determination, sample was dissolved in methanol-dimethylformamide $(7: 1 \mathrm{v} / \mathrm{v})$ and detected at $430 \mathrm{~nm}$. Notably, brown centrifugal tubes were used during the whole procedure to avoid carotenoids degradation in light.

\section{RESULTS AND DISCUSSION}

\section{Bypath Disruption for Adequate Precursors}

To increase the pools of the key precursor acetyl-CoA, some bypaths to convert acetyl-CoA into undesired products was attempted to be disrupted in our case. Krivoruchko et al. (2015) once reported that deletion of CIT2 and MLS1 enhanced 1butanol production by improving acetyl-CoA supply via avoiding trapping acetyl-CoA GYC (Figure 1A). Accordingly, these two genes were deleted individually in our previous constructed crocetin producing strain SyBE_Sc0123C050. As shown in 


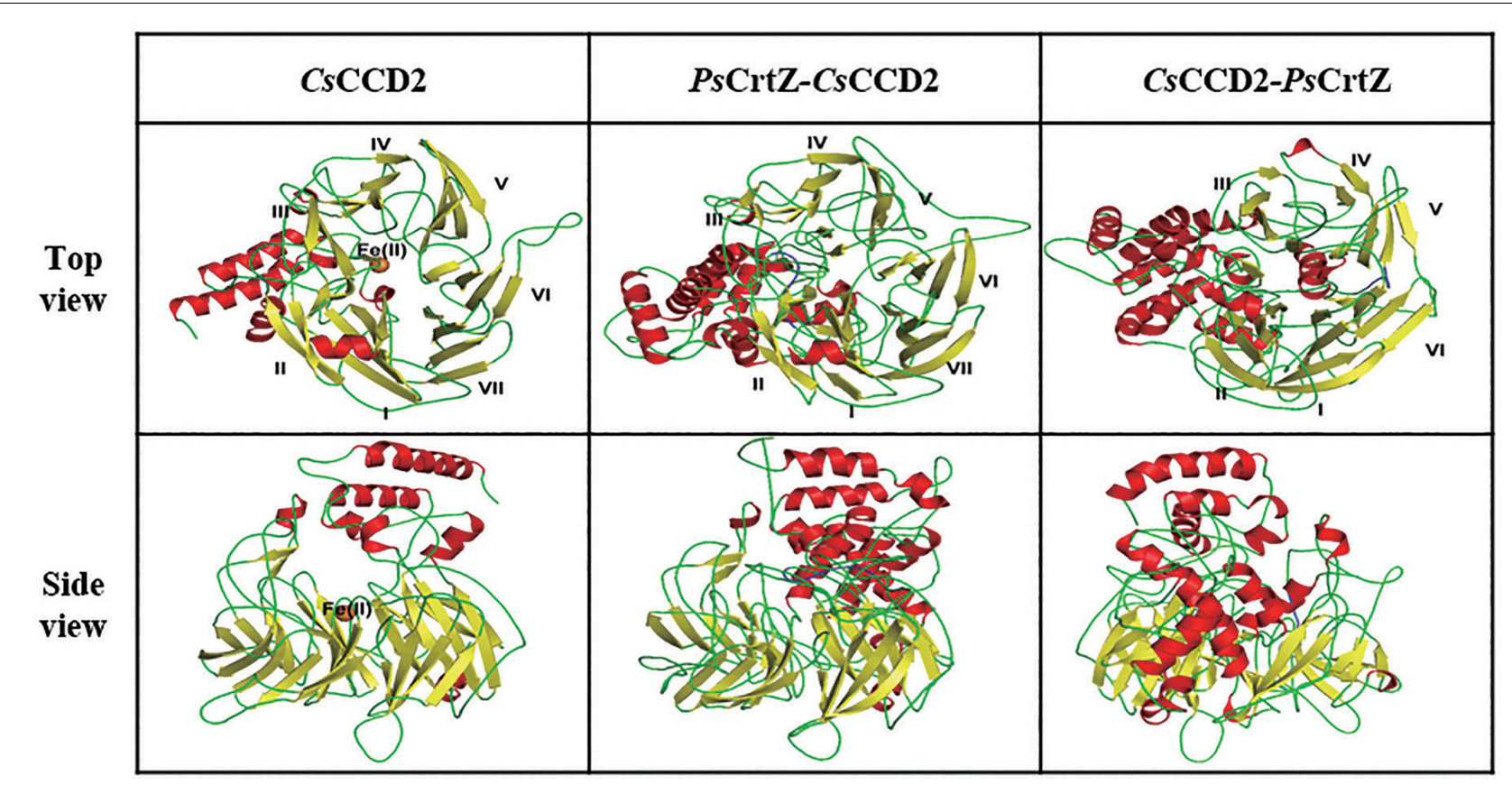

FIGURE 2 | Tridimensional structure models of the CsCCD2 (left), forward fusion protein Ps CrtZ-CsCCD2 (middle) and reverse fusion protein CsCCD2-PsCrtZ (right). The ferrous catalytic iron is colored in orange. Secondary structural elements consisting of $\alpha$-helices and $\beta$-sheets are colored in red and yellow. The blade numbers of the windmill structure are labeled.

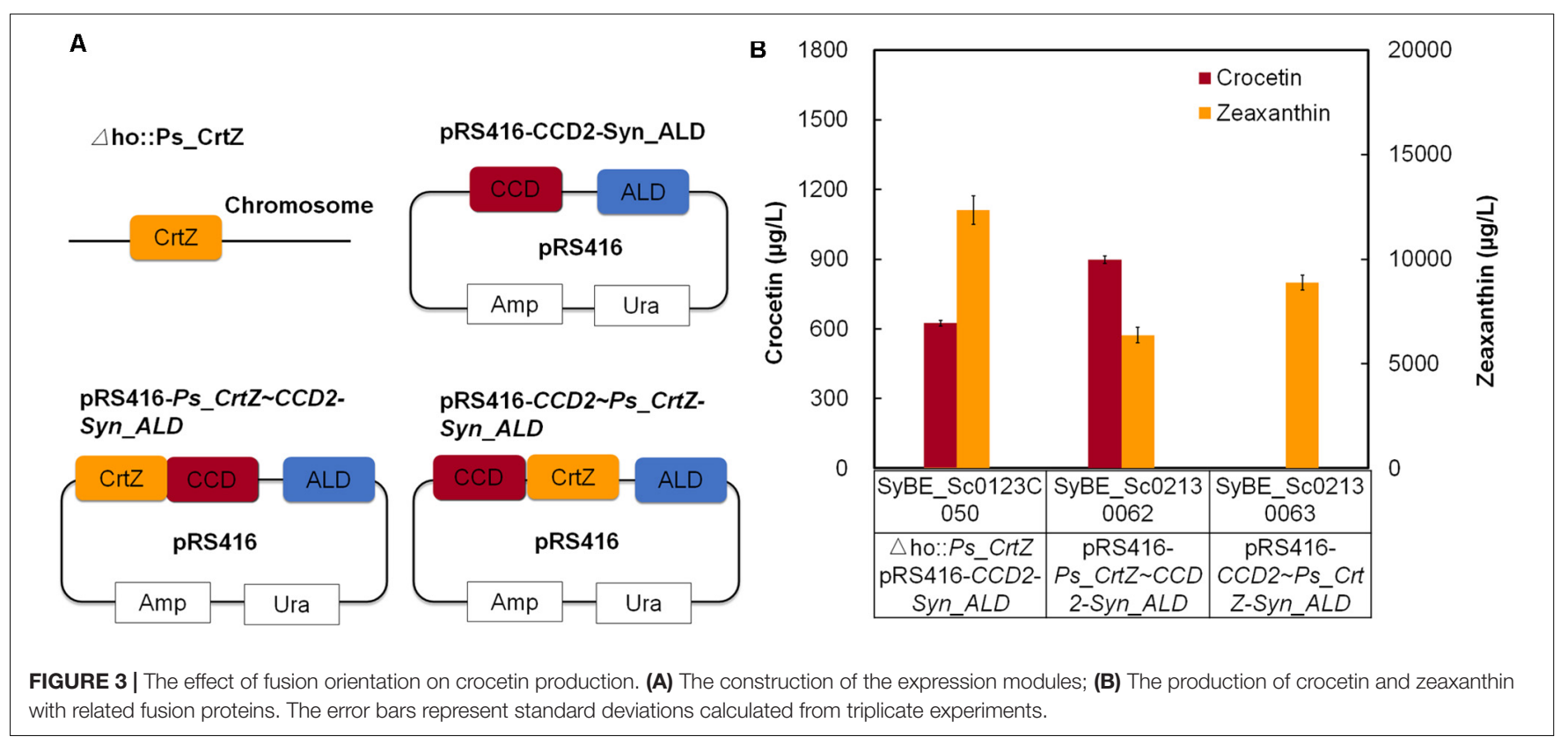

Supplementary Figure S1, individual deletion of these two genes was not resulted in growth defect under glucose. When glucose was exhausted and the carbon source was changed to ethanol, $\triangle C I T 2$ and $\triangle M L S 1$ were injurious to assimilation of ethanol to build biomass in different degrees. Chen et al. (2013) and Cobine et al. (2012) once described similar growth phenotypes of $\triangle C I T 2$ and $\triangle M L S 1$ on glucose and $2 \mathrm{C}$ carbon source (such as ethanol). Even though gene knockout decreased the final biomass, the total
acetyl-CoA content as well as the crocetin yield per DCW(g) were both significantly enhanced by individual deletion of CIT2 and $M L S 1$ (Figures 1B,C). The percent increase in the concentration of total acetyl-CoA corresponded with the enhancement in crocetin yield. Similar effect of $\triangle$ CIT2 has also been observed on other terpene production (Cobine et al., 2012). Among these approaches, $\triangle C I T 2$ achieved $49.7 \%$ improvement on total acetyl-CoA content and 1.5 times of enhancement on crocetin 
A
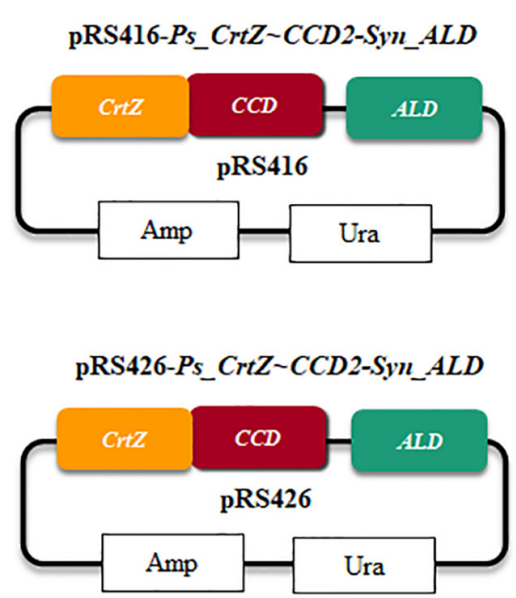

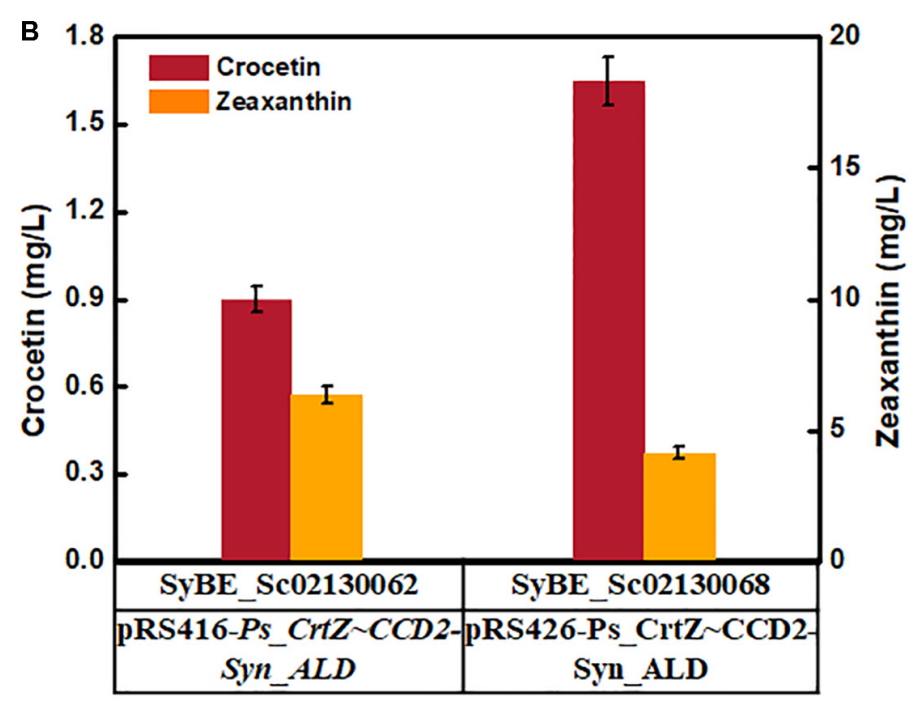

FIGURE 4 | The effect of overexpression of fused CrtZ and CCD on crocetin titer. (A) The construction of the expression modules; (B) The production of crocetin and zeaxanthin with single-copy plasmid and multicopy plasmid, respectively. The error bars represent standard deviations calculated from triplicate experiments.

yield which was the higher one than MLS1 knockout strains (Figure 1C). Therefore, CIT2 deleted strain SyBE_Sc01010682 was employed as the candidate for further optimization.

\section{Facilitation of Catalysis Efficiency of CCD2 via Suited Fusion Protein}

Since CCDs catalyzed the limiting step for crocetin synthesis (Frusciante et al., 2014), fusion expression of PsCrtZ and CsCCD2 would facilitate the enzymic catalysis efficiency of CCD2 in terms of enhancing its substrate (zeaxanthin) accessibility. In previous studies, the fusion orientation has significantly affected protein structures and even the enzyme activities. Therefore, in our study, the structure models of native CsCCD2, forward/reverse fusion of $P s \mathrm{CrtZ}$ and $\mathrm{CsCCD} 2$ were simulated and analyzed to guide our protein fusion design (Figure 2). The structural information of CCD2 showed that some non-contiguous $\alpha$-helices and loops formed the hydrophobic substrate binding tunnel on the N-terminal and a highly conserved striking "windmill" architecture consisting of seven-bladed $\beta$-propeller formed the activity center on its C-terminal (Figure 2 left). The built model of forward fusion enzyme (PsCrtZ-CsCCD2) displayed the conserved windmill architecture with seven-blade (Figure 2 middle) which was consistent with the reported structure among CCDs family (Sui et al., 2013; Frusciante et al., 2014). Therefore, the enzymic activity of CsCCD2 in forward fusion enzyme would be well retained. Moreover, the forward fusion of PsCrtZCsCCD2 shorted the distance between substrate (zeaxanthin) and N-terminal hydrophobic substrate binding tunnel of CCD2, providing an ideal environment for accumulation and availability of zeaxanthin substrates to CsCCD2. On the contrary, in the built model of reverse fusion enzyme CsCCD2-PsCrtZ, some bladed $\beta$-propeller was replaced by the allochthonous $\alpha$-helix, resulting in deletion of one of the original seven-blade (Figure 2 right). Therefore, the crucial windmill architecture of CsCCD2 was disrupted by reverse fusion enzyme CsCCD2-PsCrtZ, which would lead to inactivation of CsCCD2.

Accordingly, the forward and reverse fusion of $\mathrm{PsCrtZ}$ and CsCCD2 (SyBE_Sc02130062 and SyBE_Sc02130063) were constructed (Figure 3A), and their effects on crocetin production were further investigated. As shown in Figure 3B, comparing with the crocetin titer in stain SyBE_Sc0123C050 with two unfused enzymes, the crocetin production was increased to 1.44 times by the forward fusion (PsCrtZ-CsCCD2), while no crocetin but an amount of zeaxanthin was detected in the strain SyBE_Sc01010685 with reverse fusion (CsCCD2-PsCrtZ). These results powerfully supported our hypothesis based on the structural analysis in silico. No conversion from zeaxanthin to crocetin suggested that CsCCD2 in the reverse fusion enzyme CsCCD2-PsCrtZ was inactivated, which might due to the CsCCD2 conformational change caused by reverse fusion version. Thus, this study is another successful case of linking up structural simulation with experimental test, which provided another reference to guide protein fusion except for our previous works (Jiang et al., 2017).

As the forward fusion protein PsCrtZ-CsCCD2 was proved to be more benefit for crocetin accumulation, thus its copy number was further raised via expressing the fusion protein by multicopy plasmid pRS426 instead of single-copy plasmid pRS416 (Figure 4A). As shown in Figure 4B, crocetin production was increased by $83 \%$ compared with the one achieved by single copy expression. Therefore, strain SyBE_Sc02130068 was used to process optimization.

\section{Crocetin Production Promoted by Medium Optimization}

Through pathway engineering and heterologous protein expression, especially after CIT2 gene was knocked out, the growth has been significantly affected, indicating the 

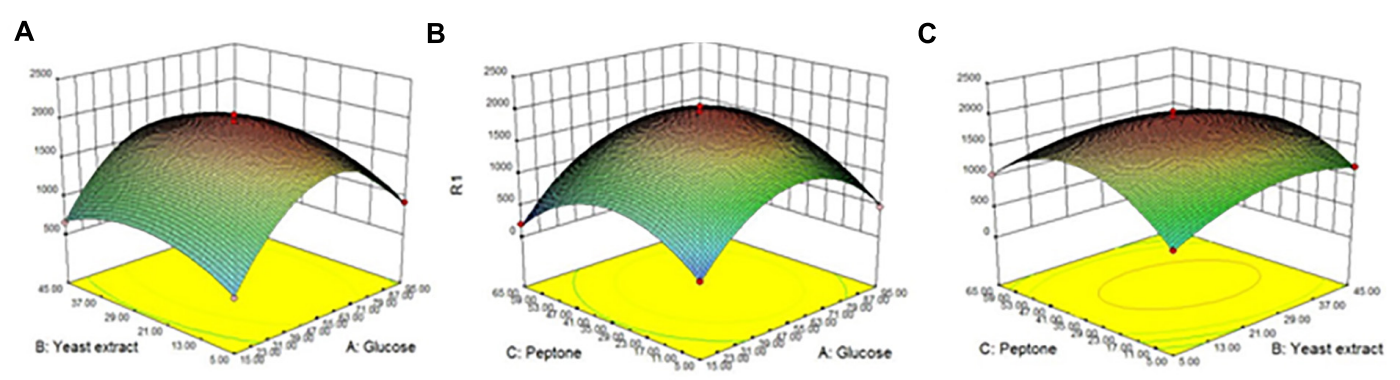

D

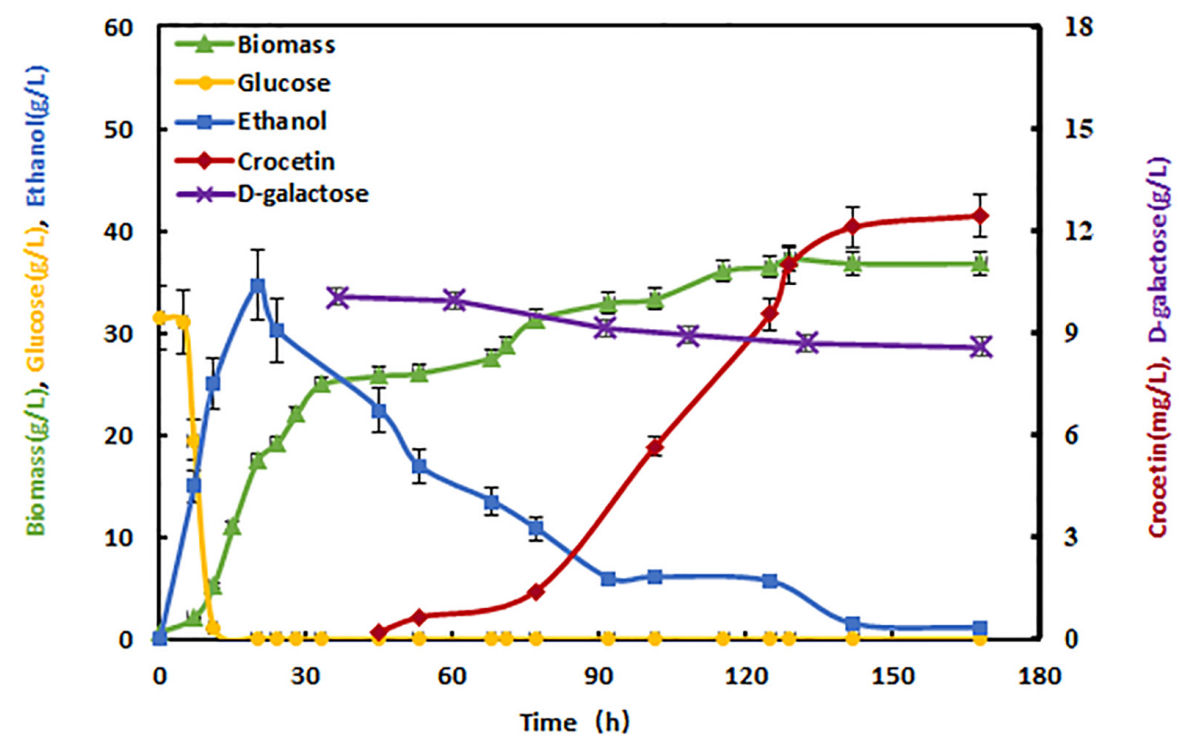

FIGURE 5 | Crocetin production promoted by medium optimization and fed-batch fermentation. The 3D surface plots of each two factors' interaction effect on the yield of crocetin. (A) The interaction effect of glucose and yeast extract on the crocetin yield; (B) The interaction effect of glucose and peptone on the crocetin yield; (C) The interaction effect of yeast extract and peptone on the crocetin yield; (D) Crocetin production in fed-batch fermentation. Crocetin production (red line), glucose (yellow line), ethanol (blue line), D-galactose (purple line), and biomass (green line) were measured during fed-batch fermentation. D-galactose was added at $36 \mathrm{~h}$. The error bars represent standard deviation calculated from duplicate experiments.

endogenous metabolism flux of the engineered strain would change to some extent. In order to adapt to these changes, it is necessary to optimize the culture medium accordingly. Medium optimization was performed using the highest crocetin yield strain SyBE_Sc02130068. Firstly, PlackettBurman (Ahuja et al., 2004) two-level fractional factorial design was used to verify the main effects among seven conventional ingredients (Supplementary Table S3). The regression analysis showed that the order of significance of the factor effects on crocetin production is: glucose $=$ yeast extract $>$ peptone $>\mathrm{CaCl}_{2}>\mathrm{KH}_{2} \mathrm{PO}_{4}>\left(\mathrm{NH}_{4}\right)_{2} \mathrm{SO}_{4}$ (Supplementary Tables S4, S5). The main effect $P$ values of glucose, yeast extract and peptone were less than 0.05 , indicating that they were the main factors. Besides, these three factors were all have positive effects on output ( $T$ value is positive). Therefore, they were tested for further optimization by steepest ascent method and the results were shown in Supplementary Table S6. With the increase of the concentration of glucose, peptone, and yeast extract, the yield of crocetin was increased firstly and then decreased at certain points. As the yield of crocetin in the group
3 was the largest one, showing that the value of each factor in this group was close to the maximal response value region. Therefore, the factor value of the group 3 was taken as the central point of the follow-up Box-Behnken RSM optimization (Supplementary Table S7) (Song et al., 2017). The experimental results were listed in Supplementary Table S8. The 3D surface plots of each two factors' interaction effect on the yield of crocetin were shown in Figures 5A-C. Through regression fitting analysis, a quadratic response surface regression model was established, and the fitting quadratic regression equation was obtained as belows:

$$
\begin{gathered}
R=1555.83462+62.78675 A+53.76462 B+56.78296 C \\
-0.032852 A B+0.051083 A C-0.25913 B C-0.54415 A 2 \\
-0.81730 B 2-0.76603 C 2
\end{gathered}
$$

In this equation, $\mathrm{R}, \mathrm{A}, \mathrm{B}$, and $\mathrm{C}$ represented crocetin production, glucose concentration, yeast extract concentration, 
and peptone concentration, respectively. Through this equation, the optimal medium formulation was obtained with $58.54 \mathrm{~g} / \mathrm{L}$ glucose, $26.24 \mathrm{~g} / \mathrm{L}$ yeast extract, $34.56 \mathrm{~g} / \mathrm{L}$ peptone, and the theoretical prediction of the maximum yield was $1.97 \mathrm{mg} / \mathrm{L}$ accordingly. The verification experiment was also done with this predicted concentration of medium ingredients, and the actual crocetin production was $1.95 \pm 0.23 \mathrm{mg} / \mathrm{L}$, which matched the prediction model very well. The correctness between the prediction and experimental results verified the credibility of the quadratic response surface regression model. The crocetin titer with the optimized fermentation medium increased by $18.5 \%$ compared to the traditional YPD medium.

\section{Crocetin Output Further Enhanced by Fed-Batch Fermentation}

To further promote the crocetin production, the engineered strain SyBE_Sc02130068 was selected for fed-batch fermentation in 5-L fermentor using the optimized medium above. As shown in Figure 5D, the fermentation process was divided into two stages. The first stage was cell growth stage in which the cultivation temperature was controlled at $30^{\circ} \mathrm{C}$ for $35 \mathrm{~h}$ until the cell density $\mathrm{OD}_{600}$ reached 105 . The supplemental carbon source glucose was strictly controlled below $1 \mathrm{~g} / \mathrm{L}$ due to carbon source restriction strategy. The second stage crocetin producing stage, was initialized after the temperature was decreased to $20^{\circ} \mathrm{C}$ and $10 \mathrm{~g} / \mathrm{L} \mathrm{D}-(+)$-galactose was added at $36 \mathrm{~h}$. The supplemental carbon source was changed into ethanol, because the existence of glucose would inhibit the expression of the GAL promoters. The ethanol concentration was maintained at nearly $5 \mathrm{~g} / \mathrm{L}$ until fermentation end. After $70 \mathrm{~h}$, the crocetin production increased exponentially until $124 \mathrm{~h}$. It was also noticed that while the crocetin accumulated sharply, the cell growth did not increase significantly. Thus, increasing the crocetin resistance in yeast will be a promising solution for crocetin further improvement. After $160 \mathrm{~h}$ cultivation, the crocetin titer reached $12.43 \pm 0.62 \mathrm{mg} / \mathrm{L}$ (Figure 5D), which was eight times more than the output in shake flask level. As shown in Figure 5D, it is found that galactose concentration decreased with increasing concentration of crocetin. And the galactose consumption might affect the production of crocetin. The absolute titer $(12.43 \mathrm{mg} / \mathrm{L})$ and the production yield based on ethanol consumption $\left(Y_{\mathrm{P} / \mathrm{S}}=0.017 \%\right)$ were still far away from commercialization level, strain development by metabolic engineering and synthetic biology to increase the product accumulation rate and product resistance, as well as process optimization for higher cell density fermentation would be basic and efficient solutions. In addition, together with off-gas analysis, involving more on-line parameters associated with growth and production as feedback will also be helpful for precise control in fermentation (Sui et al., 2016), leading to higher crocetin production.

\section{CONCLUSION}

Further enhancing the accumulation of natural products with complex structure in microbes is always a big challenge. Here, a systematic optimization was executed for crocetin overproduction in $S$. cerevisiae, mainly based on promoting precursor pools, tuning key enzymes as well as optimizing bioprocess. Through above optimizations, the crocetin production in final strain SyBE_Sc02130068 was stepwise increased by $212 \%$ compared to that in the initial strain SyBE_Sc0123C050 at shake-flask level. Finally, a titer of $12.43 \pm 0.62 \mathrm{mg} / \mathrm{L}$ crocetin was realized by 5 -L fed-batch fermentation, which was the highest crocetin titer reported in micro-organisms to date. This study not only set a good example to guide audience how to stepwise increase heterologous natural products accumulated in microbials, but also highlights the synergy between precursor engineering and key enzymes engineering is crucial for obtaining microbial overproduction of natural products.

\section{DATA AVAILABILITY STATEMENT}

All datasets presented in this study are included in the article/Supplementary Material.

\section{AUTHOR CONTRIBUTIONS}

TS and MY conceived the study. NW and CW participated in strain construction. MY and NW carried out the protein analysis. TS and WX participated in fed-batch fermentation. TS, CW, and FC carried out the chemical analysis. MD, YW, and XL helped to draft the manuscript. YY participated in design and coordination of the study. MY supervised the whole research and revised the manuscript. All the authors read and approved the final manuscript.

\section{FUNDING}

This work was supported by the funds of New Drug Creation Manufacturing Program (2019ZX09J19105), the National Key Research and Development Program of China (2018YFA0900702), and the National Natural Science Foundation of China (21621004 and 21676192).

\section{ACKNOWLEDGMENTS}

The authors are grateful to Drs. Min Dong and Yunzi Luo from Tianjin University and thank them for their kind discussion.

\section{SUPPLEMENTARY MATERIAL}

The Supplementary Material for this article can be found online at: https://www.frontiersin.org/articles/10.3389/fbioe.2020. 578005/full\#supplementary-material 


\section{REFERENCES}

Ahuja, S. K., Ferreira, G. M., and Moreira, A. R. (2004). Application of PlackettBurman design and response surface methodology to achieve exponential growth for aggregated shipworm bacterium. Biotechnol. Bioeng. 85, 666-675. doi: 10.1002/bit. 10880

Bathaie, S. Z., Hoshyar, R., Miri, H., and Sadeghizadeh, M. (2013). Anticancer effects of crocetin in both human adenocarcinoma gastric cancer cells and rat model of gastric cancer. Biochem. Cell Biol. 91, 397-403. doi: 10.1139/bcb-20130014

Boeke, J. D., LaCroute, F., and Fink, GR. (1984). A positive selection for mutants lacking orotidine-5'-phosphate decarboxylase activity in yeast: 5-fluoro-orotic acid resistance. J. Mol. Gen. Genet. 197, 345-346. doi: 10.1007/bf00330984

Chai, F., Wang, Y., Mei, X., Yao, M., Chen, Y., Liu, H., et al. (2017). Heterologous biosynthesis and manipulation of crocetin in Saccharomyces cerevisiae. Microb. Cell Fact. 16:54. doi: 10.1186/s12934-017-0665-1

Chen, Y., Daviet, L., Schalk, M., Siewers, V., and Nielsen, J. (2013). Establishing a platform cell factory through engineering of yeast acetyl-CoA metabolism. Metab. Eng. 15, 48-54. doi: 10.1016/j.ymben.2012.11.002

Cobine, P., Chen, Y., Siewers, V., and Nielsen, J. (2012). Profiling of cytosolic and peroxisomal acetyl-CoA metabolism in Saccharomyces cerevisiae. PLoS One 7:e0042475. doi: 10.1371/journal.pone.0042475

Delano, W. L., and Bioinformatics. (2002). The PyMol molecular graphics system. Proteins Struct. Funct. Bioinforma. 30, 442-454.

Deng, Y., Sun, M., Xu, S., and Zhou, J. (2016). Enhanced (S)-linalool production by fusion expression of farnesyl diphosphate synthase and linalool synthase in Saccharomyces cerevisiae. J. Appl. Microbiol. 121, 187-195. doi: 10.1111/jam. 13105

Frusciante, S., Diretto, G., Bruno, M., Ferrante, P., Pietrella, M., Prado-Cabrero, A., et al. (2014). Novel carotenoid cleavage dioxygenase catalyzes the first dedicated step in saffron crocin biosynthesis. Proc. Natl. Acad. Sci. U.S.A. 111, 12246-12251. doi: 10.1073/pnas.1404629111

Gietz, R. D. (2006). Yeast transformation by the LiAc/SS carrier DNA/PEG method. Methods Mol. Biol. 313, 107-120. doi: 10.1385/1-59259-958-3:107

Jiang, G. Z., Yao, M. D., Wang, Y., Zhou, L., Song, T. Q., Liu, H., et al. (2017). Manipulation of GES and ERG20 for geraniol overproduction in Saccharomyces cerevisiae. Metab. Eng. 41, 57-66. doi: 10.1016/j.ymben.2017.03.005

Krivoruchko, A., Zhang, Y., Siewers, V., Chen, Y., and Nielsen, J. (2015). Microbial acetyl-CoA metabolism and metabolic engineering. Metab. Eng. 28, 28-42. doi: 10.1016/j.ymben.2014.11.009

Kuntal, B. K., Aparoy, P., and Reddanna, P. (2010). EasyModeller: a graphical interface to modeller. BMC Res. Notes 3:226. doi: 10.1186/1756-0500-3-226

Lee, D., Lloyd, N. D., Pretorius, I. S., and Borneman, A. R. (2016). Heterologous production of raspberry ketone in the wine yeast Saccharomyces cerevisiae via pathway engineering and synthetic enzyme fusion. Microb. Cell Fact. 15:49. doi: 10.1186/s12934-016-0446-2

Lv, X., Wang, F., Zhou, P., Ye, L., Xie, W., Xu, H., et al. (2016). Dual regulation of cytoplasmic and mitochondrial acetyl-CoA utilization for improved isoprene production in Saccharomyces cerevisiae. Nat. Commun. 7:12851. doi: 10.1038/ ncomms 12851

Moradzadeh, M., Sadeghnia, H. R., Tabarraei, A., and Sahebkar, A. (2018). Antitumor effects of crocetin and related molecular targets. J. Cell Physiol. 233, 2170-2182. doi: $10.1002 / j c p .25953$
Nassiri-Asl, M., and Hosseinzadeh, H. (2015). "Neuropharmacology effects of saffron (Crocus sativus) and its active constituents," in Bioactive Nutraceuticals and Dietary Supplements in Neurological and Brain Disease, ed. V. R. Preedy (San Diego, CA: Academic Press), 29-39. doi: 10.1016/b978-0-12-411462-3. 00003-5

Ro, D. K., Paradise, E. M., Ouellet, M., Fisher, K. J., Newman, K. L., Ndungu, J. M., et al. (2006). Production of the antimalarial drug precursor artemisinic acid in engineered yeast. Nature 440, 940-943. doi: 10.1038/nature 04640

Sabourin, M., Tuzon, C. T., Fisher, T. S., and Zakian, V. A. (2007). A flexible protein linker improves the function of epitope-tagged proteins in Saccharomyces cerevisiae. Yeast 24, 39-45. doi: 10.1002/yea.1431

Song, T. Q., Ding, M. Z., Zhai, F., Liu, D., Liu, H., Xiao, W. H., et al. (2017). Engineering Saccharomyces cerevisiae for geranylgeraniol overproduction by combinatorial design. Sci. Rep. 7:14991. doi: 10.1038/s41598-017-15 $005-4$

Sui, X., Kiser, P. D., Lintig, J., and Palczewski, K. (2013). Structural basis of carotenoid cleavage: from bacteria to mammals. Arch Biochem. Biophys. 539, 203-213. doi: 10.1016/j.abb.2013.06.012

Sui, X., Zhang, J., Golczak, M., Palczewski, K., and Kiser, P. D. (2016). Key residues for catalytic function and metal coordination in a carotenoid cleavage dioxygenase. J. Biol. Chem. 291, 19401-19412. doi: 10.1074/jbc.M116.74 4912

Wang, W., He, P., Zhao, D., Ye, L., Dai, L., Zhang, X., et al. (2019). Construction of Escherichia coli cell factories for crocin biosynthesis. Microb. Fact. 18:120. doi: 10.1186/s12934-019-1166-1

Xiang, M., Yang, R., Zhang, Y., Wu, P., Wang, L., Gao, Z., et al. (2017). Effect of crocetin on vascular smooth muscle cells migration induced by advanced glycosylation end products. Microvasc. Res. 112, 30-36. doi: 10.1016/j.mvr.2017. 02.004

Yu, K., Liu, C., Kim, B. G., and Lee, D. Y. (2015). Synthetic fusion protein design and applications. Biotechnol. Adv. 33, 155-164. doi: 10.1016/j.biotechadv.2014. 11.005

Zhao, F., Bai, P., Liu, T., Li, D., Zhang, X., Lu, W., et al. (2016). Optimization of a cytochrome $\mathrm{P} 450$ oxidation system for enhancing protopanaxadiol production in Saccharomyces cerevisiae. Biotechnol. Bioeng. 113, 1787-1795. doi: 10.1002/ bit. 25934

Zhou, Y. J., Gao, W., Rong, Q., Jin, G., Chu, H., Liu, W., et al. (2012). Modular pathway engineering of diterpenoid synthases and the mevalonic acid pathway for miltiradiene production. J. Am. Chem. Soc. 134, 3234-3241. doi: 10.1021/ ja2114486

Conflict of Interest: The authors declare that the research was conducted in the absence of any commercial or financial relationships that could be construed as a potential conflict of interest.

Copyright (c) 2020 Song, Wu, Wang, Wang, Chai, Ding, Li, Yao, Xiao and Yuan. This is an open-access article distributed under the terms of the Creative Commons Attribution License (CC BY). The use, distribution or reproduction in other forums is permitted, provided the original author(s) and the copyright owner(s) are credited and that the original publication in this journal is cited, in accordance with accepted academic practice. No use, distribution or reproduction is permitted which does not comply with these terms. 